\title{
Photon: history, mass, charge *
}

\author{
L.B. Okun \\ 117218 ITEP, Moscow, Russia
}

February 2, 2008

\begin{abstract}
The talk consists of three parts. "History" briefly describes the emergence and evolution of the concept of photon during the first two decades of the 20th century. "Mass" gives a short review of the literature on the upper limit of the photon's mass. "Charge" is a critical discussion of the existing interpretation of searches for photon charge. Schemes, in which all photons are charged, are grossly inconsistent. A model with three kinds of photons (positive, negative and neutral) seems at first sight to be more consistent, but turns out to have its own serious problems.
\end{abstract}

\section{History}

The idea that light consists of rapidly moving particles can be traced from the writings of ancient authors to Descartes and Newton. The wave theory of light was put forward by Huyghens and was later decisively proved to be correct through discovery of interference and diffraction by Young and Fresnel. Maxwell's theory of light as electromagnetic waves was one of the greatest achievements of the 19th century.

The history of the photon in the 20th century started in 1901 with the formula by Planck for radiation of a black body and introduction of what was called later the quantum of action $h$ [1]. In 1902 Lenard discovered that energy of electrons in photoeffect does not depend on the intensity of light, while it depends on the wavelength of the latter [2].

In his fundamental article "On an euristic point of view concerning the production and transformation of light" published in 1905 Einstein pointed out that the discovery of Lenard meant that energy of light is distributed in space not uniformly, but in a form of localized light quanta $[3$. He has shown that all experiments related to the black body radiation, photoluminescence and production of cathode rays by ultraviolet light can be explained by the quanta of light.

*Presented at the International Conference on the Structure and Interactions of the Photon "Photon 2005: its first hundred years and the future", Warsaw University, 31.08.-04.09.2005 
The proof that Einstein's light quanta behave as particles, carrying not only energy, but also momentum, was given in 1923 in the experiments by Compton on scattering of X-rays on electrons 4 .

The term "photon" for particles of light was coined by Lewis in 1926 in an article "The conservation of photons" [5]. His notion of a photon was different from the notion we use today. He considered photons to be "atoms" of light, which analogously to the ordinary atoms are conserved.

The term "photon" was quickly accepted by physics community. The fifth Solvay Council of physics, which took place on October 24-29, 1927, had the name "Electrons and Photons" [6]. The term "photon" in its present meaning was first used in the talk by Compton at this meeting (see ref. [6], p. 55).

In his talk Compton used the term "photon" as if it existed since 1905; thus on page 62 of ref. [6] one can read: "It is known that the hypothesis of photons was introduced by Einstein in order to explain the photo-electric effect". On the other hand, on page 57 one can read:

"When speaking of this unit of radiation, I would use the name "photon" suggested recently by G.N. Lewis (Nature, 18 December, 1926). ... it has the advantage of being brief without implying any relation with mechanics of quanta, more general, or the quantum theorie of atomic structure".

The Proceedings [6] open with an obituary of H.A. Lorentz who passed away in February 1928, a few months after the Fifth Solvay meeting, in which Lorentz actively participated.

The speakers at the meeting were:

W.-L. Bragg, The intensity of reflected X-rays, pp. 1-44;

A.H. Compton, Discordances between the experiment and the electromagnetic theory of radiation, pp. 55-86;

L. de Broglie, The new dynamics of quanta, pp. 105-133;

M. Born and W. Heisenberg, The mechanics of quanta, pp. 143-182;

E. Schrödinger, The mechanics of waves, pp. 185-207;

N. Bohr, The postulate of quanta and the development of atomistics, pp. 215-248.

Each of the talks was followed by a detailed discussion. Participated Bohr, Born, Brillouin, de Broglie, Compton, Dirac, De Donder, Ehrenfest, Fowler, Heisenberg, Kramers, Langmuir, Langevin, Lorentz, Pauli, Richardson, Schrödinger.

Einstein took part only in the "General discussion of the new ideas", expressed during the meeting. The discussion (pp. 248-289) was presented in three sections: 1. Causality, Determinisme, Probability; 2. Photons; 3. Photons and Electrons.

Einstein spoke in the first section (pp. 253-256) and asked a question during the second section (p. 266). He considered a screen with a small hole in it and a spherical layer of photoemulsion of large radius behind it. Electrons fall on the screen as De Broglie - Schrödinger plane waves normal to it and reach the emulsion as spherical waves. Einstein discussed the two possible interpretations of this thought experiment: purely statistical and purely deterministic. The term "photon" was not used in his remarks. 
The term "photon" was again used by Compton on December 12, 1927, this time without any reference to Lewis, in Compton's Nobel lecture "X-rays as a branch of optics" 7]. On page 186 one can read:

"An X-ray photon is deflected through an angle $\phi$ by an electron, which in turn recoils at an angle $\theta$, taking a part of the energy of photon".

Further on page 187:

"... recoil electrons are in accord with the predictions of the photon theory".

The year 1927 marked the end of the history of emergence of the concept of photon. A few years later Dirac opened a new chapter in Physics by establishing Quantum Electrodynamics.

As for Einstein, he wrote in 1951:

"All these fifty years of pondering have not brought me any closer to answering the question, What are light quanta?" 8].

\section{Mass}

The problem of the upper limit on the mass of the photon was raised at ITEP by Isaak Yakovlevich Pomeranchuk (20.05.1913 - 14.12.1966) in autumn of 1966, a few months before he lost his fight against cancer. He put this question to his former students: Igor Yuryevich Kobzarev (15.10.1932 - 20.01.1991) and myself. First we wrote a draft of a short research note, but then after a thorough search we discovered that most of our considerations had been already addressed in the literature by de Broglie [9], 10] (see also [11, [12]), Schrödinger [13], 14], Bass and Schrödinger [15] and by Gintsburg [16].

In particular, de Broglie [10 noticed that photon mass would lead to a faster speed of violet light than that of the red one. He concluded that during the eclipse in double star system the color of the appearing star would change from violet to red. He also considered the dispersion of radiowaves.

Schrödinger [13, 14] pointed out that magnetic field of the Earth would be exponentially cut off at distances of the order of the photon Compton wave length $\lambda_{\gamma}=1 / m_{\gamma}$. From the observed altitude of auroras he concluded that $\lambda_{\gamma}>10^{4} \mathrm{~km}$.

Gintsburg [16] corrected the limit of Schrödinger and suggested that measurements of the magnetic field of Jupiter could improve the limit to $\lambda_{\gamma} \sim 10^{6}$ $\mathrm{km}$. He also was the first to consider how the mass of the photon would influence the magnetohydrodynamic waves in plasma.

These results discouraged us from publishing an original article.

From the beginning of 1968 a special issue of Uspekhi Fizicheskikh Nauk was under preparation to mark 50 years of this review journal. Kobzarev and I were invited to publish our paper on photon as a review.

In this short review [17] we corrected the estimates by de Broglie and Schrödinger. The former estimate was invalidated due to dispersion of light in the atmospheres of stars (we found that this effect was considered by Lebedev in 1908 [18]). Ref. [18] was the paper, which closed the discussion of colour 
variation in binary stars. The effect was discovered by Belopolskii 19, Nordmann [20] and Tikhoff [21] and interpreted by them as dispersion of light in the interstellar free space. The observed minimum in red light preceded that in violet light from the variable binary stars by a few minutes. (Note that for a massive photon the violet light should be faster, not slower!) Lebedev ${ }^{1}$ rejected this interpretation and explained the effect by the difference of pressure in the atmospheres of two stars [22, 23].

We also found that a much better limit could be extracted from the measurements by Mandelstam 24] of radiowave dispersion in the atmosphere of the Earth.

As for the limit by Schrödinger we conservatively extended it to $30000 \mathrm{~km}$ by using the data from review by Bierman [25], though these data (from rockets and satellites) indicated the spread of the geomagnetic field to $60000 \mathrm{~km}$ and even to $100000 \mathrm{~km}$.

In addition to magnetic field we have interpreted in terms of $\lambda_{\gamma}$ the experiment by Plimpton and Lawton [26, testing the absence of Coulomb field in the space between two concentric spheres, and derived $\lambda_{\gamma}<10 \mathrm{~km}$. (The deviation from Coulomb law was parametrized in ref. [26] by $1 / r^{2+\varepsilon}$.)

We also discussed why the longitudinal photons do not manifest themselves in the black body radiation, a subject considered by Bass and Schrödinger [15].

Our review [17 appeared in May 1968.

Two months later Physical Review Letters received and in August published a paper by Goldhaber and Nietto 27] "New geomagnetic limit on the mass of the photon". Their geomagnetic limit was about $90000 \mathrm{~km}$. They derived $\lambda_{\gamma}<10 \mathrm{~km}$ from reference [26] and reconsidered the geomagnetic estimates by Gintsburg [16].

Three years later Goldhaber and Nietto published an extensive review 28] with about 100 references. The review by Byrne 29 published in 1977 has about 40 references. The latest review by Tu, Luo and Gillies [30 published in 2005 has about 200 references.

It is impossible to comment on all these hundreds of papers in a short review. One has to make selection.

Since 1992 the selected references on the photon mass are cited by the Particle Data Group (PDG) in biennial Reviews of Particle properties [31 - 37. The best cited limits (in eV) were chosen by PDG:

1992: $3 \cdot 10^{-27}$, Chibisov 38, galactic magnetic field. 1994: $3 \cdot 10^{-27}$, Chibisov 38, galactic magnetic field. 1996: $6 \cdot 10^{-16}$, Davis et al. [39], Jupiter magnetic field. 1998: $2 \cdot 10^{-16}$, Lakes [40, torque on toroid balance. 2000: $2 \cdot 10^{-16}$, Lakes 40], torque on toroid balance. 2002: $2 \cdot 10^{-16}$, Lakes [40], torque on toroid balance. 2004: $6 \cdot 10^{-17}$, Ryutov [41], magnetohydrodynamics of solar wind (MHD).

\footnotetext{
${ }^{1}$ Petr Nikolaevich Lebedev (1866-1912) is famous by his experimental discovery of pressure of light.
} 
If $c$ is the unit of velocity and $\hbar$ is the unit of action, then $1 \mathrm{eV}=1.78 \cdot 10^{-33}$ g., $1 \mathrm{eV}=\left(1.97 \cdot 10^{-10} \mathrm{~km}\right)^{-1}$.

Chibisov [38] considered the conditions of equilibrium of magnetic field in the smaller Magellanic cloud by applying virial theorem. This gave $\lambda_{\gamma} \lesssim l$, where $l$ is the size of the cloud $\left(l \simeq 3 \mathrm{kpc}=3 \cdot 3.08 \cdot 10^{16} \mathrm{~km} \approx 10^{17} \mathrm{~km}=10^{22} \mathrm{~cm}\right)$. It is not clear how reliable is this approach.

Davis et al. [39] used the "Jupiter suggestion" of Gintsburg [16] and the new Pioneer-10 data on the magnetic field of Jupiter.

A novel idea was put forward and realized by Lakes [40]. He exploited the fact that the term $m_{\gamma}^{2} A^{2}$ in Lagrangian breaks the gauge invariance of Maxwell's electrodynamics. In Lorenz ${ }^{2}$ gauge one has the Maxwell-Proca equation. As a result the vector potential A becomes observable. Lakes performed an experiment with a toroid Cavendish balance to search for the torque $m_{\gamma}^{2} A$ produced by the ambient vector potential $A$.

The experiment [40] disclosed that $A m_{\gamma}^{2}<2 \cdot 10^{-9} \mathrm{Tm} / \mathrm{m}^{2}$. If the cosmic vector potential $A$ is $10^{12} \mathrm{Tm}$, then $\lambda_{\gamma}=m_{\gamma}^{-1} \gtrsim 2 \cdot 10^{10} \mathrm{~m}$. This limit has been improved by other authors (see ref. [30]). However the estimate of the value of cosmic potential $A$ is not reliable enough.

Ryutov [41] developed the idea of Gintsburg [34] and first derived a selfconsistent and complete set of MHD equations accounting for finite photon mass. He did not put a new limit on the photon mass, but mentioned a possible way of improving it by the analysis of the sector structure of the Solar wind. In particular he noticed that the limit $6 \cdot 10^{-16} \mathrm{eV}$, considered in 1996 by PDG as the best one should be reduced by approximately an order of magnitude. This is the origin of the PDG best number in 2004.

\section{Charge}

There exist about a dozen of papers [43] - [52] questioning the neutrality of photons and setting an upper limit on their charge. In all of them the upper limit follows from the non-observation of any action of external static electric or magnetic fields on photon's charge, while the fact that these fields themselves are "built from photons" is ignored. As a result all those papers [43 - 52 lack a self-consistent phenomenological basis. But without such a basis any interpretation of experimental data is meaningless.

In fact the authors 43 - 52 implicitly assumed that all photons are either neutral as in ordinary QED, or all are charged. It is obvious that the latter assumption is impossible to reconcile with the existence of classical static electric or magnetic fields. Hence the best upper limit on the value of photon charge presented by the Particle Data Group [45] seems to be meaningless.

It is clear, that for a more consistent interpretation of searches [43 - 52] both types of photons are necessary: charged and neutral. In such a scheme classical electric and magnetic fields are built from the latter. Hence the scattering of

\footnotetext{
${ }^{2}$ Quite often the Lorenz gauge is erroneously ascribed to Lorentz (for clarification and for earlier references see ref. [42]).
} 
all charged particles (including the charged photons) by these fields occurs due to absorption of virtual neutral photons. Charge is conserved in this processes. (The failure of theoretical attempts to violate the conservation of electric charge was analyzed in references 53, 54.)

However a scheme with both charged and neutral photons is also not without serious problems. One of them is the catastrophic infrared emission of neutral photons by massless charged ones. The other problems are connected with the emission and absorption of charged photons by ordinary charged particles, say, electrons.

Conservation of charge calls in this case for the existence of a twin electron with charge $e-e^{\prime}$, where $e^{\prime}$ is the charge of the emitted charged photon, which is assumed to be much smaller than $e$. The mass of the twin must be much larger than the mass of the electron in order to avoid contradiction with data on atomic, nuclear, and high energy physics.

One might consider the three photons with charges $+e^{\prime},-e^{\prime}, 0$ as an $\mathrm{SU}(2)$ Yang-Mills triplet, while the electron with charge $e$ and its twin with charge

$e-e^{\prime}$ as an $\mathrm{SU}(2)$ doublet. The $\mathrm{SU}(2)$ symmetry requires mass degeneracy of particles belonging to the same multiplet. However even in this degenerate case it is impossible to accommodate the inequality $e^{\prime} / e \ll 1$ in a scheme without "astronomically huge" Higgs multiplets. The situation is further aggravated by the breaking of SU(2) gauge symmetry, responsible for the difference of masses of particles and their twins.

\section{Acknowledgments}

I am grateful to V.P. Vizgin, K.A. Tomilin and A.D. Sukhanov for helpful discussions on the history of the concept of photon.

I am grateful to A. Buras, V. Fadin, and P. Zerwas for drawing my attention to the articles on hypothetical charge of the photon and to G. Cocconi, G. Giudice, and M. Vysotsky for valuable remarks.

I am grateful to M. Krawczyk for wonderful hospitality.

The work was supported by the grant of the Russian ministry of education and science No. 2328.2003.2.

\section{References}

[1] M. Planck, Ann. Phys. 4 (1901) 561.

[2] P. Lenard, Ann. Phys. 8 (1902) 169.

[3] A. Einstein, Ann. Phys. 17 (1905) 132.

[4] A.H. Compton, Phys. Rev. 22 (1923) 409.

[5] G.N. Lewis, Nature, No. 2981, vol. 118 (December 18, 1926$) 874$. 
[6] Electrons et Photons. Rapports et discussions du cinquiem conseil de physique tenu a Bruxelles du 24 au 29 octobre 1927 sous les auspices de l'Institut International de Physique Solvay. Paris, Gauthier-Villars et cie Editeurs, 1928.

[7] A.H. Compton, in "Nobel Lectures. Physics. 1901-1995" CD-ROM, World Scientific, 1998.

[8] Abraham Pais, 'Subtle in the Lord ...' The Science and the Life of Albert Einstein, Section 19f, p. 382, Oxford, 1982.

[9] L. de Broglie, Phil. Mag. 47 (1924) 446.

[10] L. de Broglie, La mechanique ondulatoire du photon. Une nouvelle theorie de la lumiere, tome premier, Paris, 1940, pp. 39, 40.

[11] L. de Broglie, La mechanique ondulatoire du photon et theorie quantique de champs, Paris, 1949.

[12] L. de Broglie, La theorie generale des particule á Spin, Paris, 1943, p. 191.

[13] E. Shrödinger, Proc. Roy. Irish Acad. A49 (1943) 43.

[14] E. Shrödinger, Proc. Roy. Irish Acad. A49 (1943) 135.

[15] L. Bass, E. Shrödinger, Proc. Roy. Soc. A232 (1955) 1.

[16] M.A. Gintsburg, Astronom. Zhurnal 40 (1963) 703-709 (in Russian); M.A. Gintsburg, Sov. Astron. - AJ 7 (1964) 536-540 (English translation).

[17] I.Yu. Kobzarev, L.B. Okun, Uspekhi Fiz. Nauk 95 (1968) 131-137; I.Yu. Kobzarev, L.B. Okun, Sov. Phys. Usp. 11 (1968) 338-341.

[18] P.N. Lebedev, Sobranie sochineniy (Collected papers), M., Izd. AN SSSR, 1963, paper 33 (in Russian).

[19] A.A. Belopolskii, Izvestia Imper. Akademii Nauk 21 (1904) 153 (in Russian).

[20] C. Normann, Comptes Rendus 146 (1908) 266, 383.

[21] G.A. Tikhoff, Comptes Rendus 146 (1908) 570.

[22] P.N. Lebedev, Izv. Imp. AN 24 (1906) 93 (in Rusian).

[23] P.N. Lebedev, Comptes Rendus 146 (1908) 1254; 147 (1908) 515.

[24] L.I. Mandelshtam, Polnoe sobranie trudov (Complete works), Izv. AN SSSR, 1947, v. 2, pp. 277-305; v. 3, p. 238 (in Russian).

[25] L. Bierman, Sitzber. Bayerische Akad. Wissenschaften 37 (1965).

[26] S.J. Plimpton, W.E. Lawton, Phys. Rev. 50 (1936) 1066. 
[27] A.S. Goldhaber, M.M. Nieto, Phys. Rev. Lett. 21 (1968) 567.

[28] A.S. Goldhaber, M.M. Nieto, Rev. Mod. Phys. 43 (1971) 277.

[29] J.C. Byrne, Astrophysics and Space Science 46 (1977) 115-132.

[30] L-C. Tu, J. Luo, G.T. Gillies, Rep. Prog. Phys. 68 (2005) 77-130.

[31] PDG, Phys. Rev. D 45 (1992) V.1.

[32] PDG, Phys. Rev. D 50 (1994) 1351.

[33] PDG, Phys. Rev. D 54 (1996) 207.

[34] PDG, Eur. Phys. J. 3 (1998) 223.

[35] PDG, Eur. Phys. J. 15 (2000) 249.

[36] PDG, Phys. Rev. D 66 (2002) 281.

[37] PDG, Phys. Lett. B 592 (2004) 335.

[38] G.V. Chibisov, Usp. Fiz. Nauk 119 (1976) 551-5 (in Russian); Sov. Phys. Usp. 19 (1976) 624-6 (English translation).

[39] L. Davis, A.S. Goldhaber, M.M. Nieto, Phys. Rev. 35 (1975) 1402-5.

[40] R. Lakes, Phys. Rev. Lett. 80 (1998) 1826-9.

[41] D.D. Ryutov, Plasma Phys. Control Fusion 39 (1997) A73-A82.

[42] J.D. Jackson, L.B. Okun, Rev. Mod. Phys. 73 (2001) 663-680.

[43] L. Grodzins, D. Engelberg, W. Bertozzi, Bull. Am. Phys. Soc. 6 (1961) 63.

[44] R. W. Stover, T.I. Moran, and J.W. Trischka, Phys. Rev. 164 (1967) 1599.

[45] PDG, "Review of Particle Physics", Phys. Lett. B 592 (2004) 31, 335.

[46] G. Cocconi, Phys. Lett. B 206 (1988) 705.

[47] G. Raffelt, Phys. Rev D 50 (1994) 7729; hep - ph/0411398.

[48] G. Cocconi, Am. J. Phys. 60 (1992) 750.

[49] V.V. Kobyshev and S.B. Popov, hep- ph/0411398.

[50] C. Sivaram, Am. J. Phys. 63 (1994) 1473.

[51] C. Caprini and P.G. Ferreira, JCAP 0502 (2005) 006; hep-ph/0310066

[52] Y.K. Semertzidis, G.T. Danby, D.M. Lazarus, Phys. Rev. D 67 (2003) 017701.

[53] L.B. Okun and Ya.B. Zeldovich, Phys. Lett. B 78 (1978) 597.

[54] M.B. Voloshin and L.B. Okun, Pis'ma ZhETF 28 (1978) 156 (in Russian); JETP Lett. 28 (1978)145. 